\title{
Track-Induced Clustering in Position Sensitive Detector Characterization
}

\section{Mäenpää, Teppo}

2010

Mäenpää , T , Kortelainen , M J \& Lampen , T 2010 , ' Track-Induced Clustering in Position Sensitive Detector Characterization ' , IEEE Transactions on Nuclear Science, vol. 57 , no. 4 , pp. 2196-2199 . https://doi.org/10.1109/TNS.2010.2050905

http://hdl.handle.net/10138/18044

https://doi.org/10.1109/TNS.2010.2050905

publishedVersion

Downloaded from Helda, University of Helsinki institutional repository.

This is an electronic reprint of the original article.

This reprint may differ from the original in pagination and typographic detail.

Please cite the original version. 


\title{
Track-Induced Clustering in Position Sensitive Detector Characterization
}

\author{
Teppo Mäenpää, Matti J. Kortelainen, and Tapio Lampén
}

\begin{abstract}
The formation of clusters in the data analysis of position-sensitive detectors is traditionally based on signal-to-noise ratio thresholds. For detectors with a very low signal-to-noise ratio, e.g., as a result of radiation damage, the total collected charge obtained from the clusters is biased to the greater signal values resulting from the thresholds. In this paper an unbiased method to measure the charge collection of a silicon strip detector in a test beam environment is presented. The method is based on constructing the clusters on test detectors around the impact point of the reference track.
\end{abstract}

Index Terms-Beam test, clusterless analysis, signal-to-noise ratio, strip detector, track reconstruction.

\section{INTRODUCTION}

$\mathbf{T}$ HE luminosity upgrade of the CERN Large Hadron Collider (LHC) is one of the driving forces of the development of new radiation hard detectors. As part of this research, the performance of severely irradiated detectors needs to be measured. The final characterization of these detectors is carried out in a particle beam whose tracks are measured with reference detectors. The clustering methods that are commonly used introduce a bias which can dominate the measured characteristics of severely irradiated detectors. We present an unbiased, robust, and simple method for the characterization of position-sensitive detectors approaching the end of their operational life-time. For illustration, we apply this method to data of irradiated silicon strip detectors described in [1].

\section{Test Beam Data Analysis}

A typical test beam experiment consists of several well-operating position-sensitive reference detectors and at least one device under test (DUT). The reference detector data are used to reconstruct the reference tracks, and the interpolated track position to the DUT surface is used for the analysis of the DUT data. A brief description of a typical process for reference track reconstruction is given in Section II-A and one of that of the DUT analysis in Section II-B. More detailed descriptions can be found in e.g., in [2]. References [3]-[8] describe various set-ups utilizing a similar approach to data analysis. In addition, studies presented in [9], [10] utilize other types of testbeam analysis approaches benefitting from reconstructed tracks.

Manuscript received November 10, 2009; revised March 08, 2010; accepted May 13, 2010. Date of publication July 12, 2010; date of current version August $18,2010$.

The authors are with the Helsinki Institute of Physics, 00014 Helsinki, Finland (e-mail: teppo.maenpaa@helsinki.fi)

Color versions of one or more of the figures in this paper are available online at http://ieeexplore.iee.org.

Digital Object Identifier 10.1109/TNS.2010.2050905

\section{A. Reference Detectors}

The pedestal and noise levels of each strip can be estimated from separate calibration data, which have been recorded in the absence of particles passing the detectors. Alternatively, they can be estimated by excluding the contributions from real particles. This can be done by filtering out the channels with a high enough signal-to-noise ratio (SNR), for example.

The pedestals are then subtracted from the raw ADC values. Then a common mode is calculated for each event and read-out chip and subtracted from the data. Clusters are formed as adjacent strips, with pedestal and common-mode corrected signals exceeding some signal-to-noise thresholds. Cluster positions can be estimated with e.g., center of gravity methods. Finally, the tracks are reconstructed from the cluster positions of the reference detectors, typically as a straight line fit in the case of a negligible magnetic field.

To reconstruct tracks as precisely as possible, reference detector positions should be known to within sub-pitch accuracy. This can be achieved with e.g., track-based alignment.

\section{B. Test Detectors}

Typically, the same work flow as for the reference data is also used for the DUT data. Different thresholds may be used for clusterization. The reference tracks are interpolated or extrapolated to the DUT surface. The nearest DUT cluster to the track impact point is then considered to be the signal cluster. The collected charge from the particle impact can then be estimated from the cluster signal, e.g., as the sum of the signal of all the strips or as the maximum sum of two adjacent strips.

\section{Virtual Pedestal Runs}

The contribution of a particle-induced signal must be filtered out when the pedestal and noise levels are being estimated (Section II-A). If a SNR cut is used, also a significant fraction of the actual noise is discarded if the SNR cut is of the same magnitude as the standard deviation of the noise distribution or the noise distribution has large, non-Gaussian tails. This would lead to biased estimates of pedestals and noise levels.

Additionally, a part of a real cluster (for instance a strip with only a small fraction of the particle-induced signal) may pass the SNR cut and affect the estimation of the noise level. Separate pedestal runs (Section II-A) are usually less error-prone, but they are sensitive to environmental changes in time and require the absence of real clusters to be ensured.

The solution offered by a reference track-induced analysis is to benefit from the location of the tracks being already known. Assuming that the reference system can guarantee that all the 


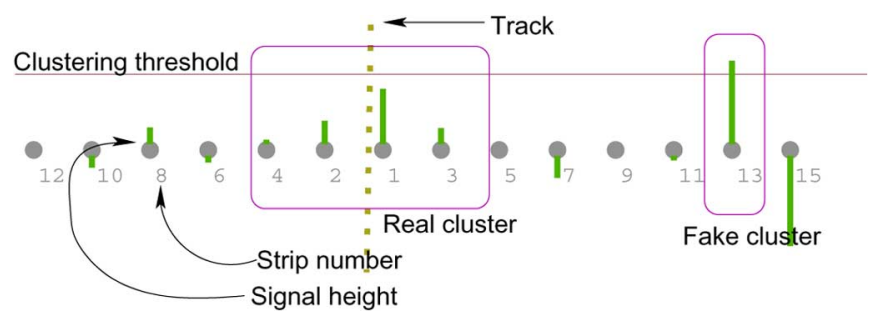

Fig. 1. Illustration of traditional and track-Induced clustering. In the traditional approach, strips with a signal value higher than the clustering threshold are selected as cluster candidates (see text for details). In this case the clustering threshold is too high to reconstruct the real cluster (left), but reconstructs a fake cluster (right). The fake cluster is also associated with the track. In track-induced clustering, a 4-strip cluster is formed around the reference track. The strip numbering corresponds to the distance from the reconstructed track.

sources of external beam-related stimulation of the detector surface have been found, for each event there is a fraction of the detector surface that can be used for pedestal, noise, and commonmode calculation in that event without the risk of biased results. This approach has advantages over the use of separate pedestal runs: 1) there is no need to record a separate pedestal run, which is more wallclock time-efficient during data-taking; 2) there is no need to ensure the absence of genuine signal(s) during pedestal runs, which might speed up the assembly of the measurement apparatus; 3 ) the pedestal information does not have to be temporally separated from the data to which it is applied, which reduces the risk of systematic errors caused by environmental changes.

While the simple approach using separate pedestal runs does reduce the risk of miscalculated pedestal and noise values, it cannot easily be used to ensure a non-biased calculation of common-mode correction.

Virtual pedestal runs can only be used when the reference system can guarantee that the fractions of DUT were not hit by particles. A reference system that does not cover the full active area of a $\mathrm{DUT}^{1}$ or has no way of indicating areas where the existence or absence of a particle-induced signal is uncertain does not fulfill the requirements and therefore should not be used for virtual pedestal runs.

\section{TRACK-INDUCED Clustering}

The traditional approach to cluster formation [2] is appropriate when the bias resulting from the use of various thresholds can be neglected. The method of track-induced clustering (TIC) avoids this bias. In track-induced clustering, the only pre-defined constant is the width of the cluster. In each event in which one track is reconstructed, a cluster is formed around the impact point of the track. As many nearest strips as are stated by the cluster width are taken into the cluster. This is depicted in Fig. 1 as "Real cluster". A comparison of the main differences between the two methods is presented in Table I.

\section{A. Control Measurement for Track-induced Clustering}

To characterize the sensor's response to a particle-induced signal, one needs to disentangle the signals generated by the

\footnotetext{
${ }^{1}$ This restriction does not apply if the absence of a beam outside the active area of the reference system can be guaranteed by other means.
}

TABLE I

COMPARISON OF TRACK-INDUCED AND TRADITIONAL CLUSTERING

\begin{tabular}{lll} 
& $\begin{array}{l}\text { Track-Induced } \\
\text { Clustering }\end{array}$ & $\begin{array}{l}\text { Traditional } \\
\text { Clustering }\end{array}$ \\
\hline Dependence & $\begin{array}{l}\text { requires a track } \\
\text { (optimized reference } \\
\text { detectors, alignment, etc.) }\end{array}$ & independent \\
Settings & cluster width & $\begin{array}{l}\text { several thresholds } \\
\text { depending of sensor's properties }\end{array}$ \\
Real Cluster & $\begin{array}{l}\text { reconstructed always } \\
\text { when track is detected }\end{array}$ & $\begin{array}{l}\text { reconstructed if } \\
\text { thresholds low enough }\end{array}$ \\
Fake Clusters & $\begin{array}{l}\text { never (only if } \\
\text { fake track detected) }\end{array}$ & $\begin{array}{l}\text { exist, some can be removed } \\
\text { with high enough thresholds }\end{array}$ \\
Association & implicit & $\begin{array}{l}\text { selection by residual or pattern matching } \\
\text { if one or more clusters are found } \\
\text { (fake cluster may be selected) }\end{array}$ \\
Method output & signal, noise & $\begin{array}{l}\text { signal, noise, resolution, efficiency, } \\
\text { clusters' predictive value [2] }\end{array}$
\end{tabular}

particle from the signals generated by noise or the measurement setup. This can be done with a control measurement carried out with the absence of crossing particles.

The control measurement can be done in the following way: the cluster of event $N$ is formed around the impact point of some other event far enough in time, for instance event $N-m$. This approach automatically takes into account the non-uniformity of the beam and avoids, as far as possible, the caveats related to time-dependent effects and the properties of the data-acquisition system (DAQ). The approach requires the beam spread to be large enough for the impact points in events $N$ and $N-m$ to be apart; this assumption can usually be made since test beams are tuned to cover as large area of the DUT as possible. If this assumption cannot be made, the problematic events need to be filtered out.

\section{B. Determination of Sensor's Response}

Detailed unbiased analysis concerning the characteristics of the DUT can be performed by comparing the properties of the clusters generated with the track-induced clustering and the corresponding control measurement. The differences are generated solely by the passing particles.

\section{RESULTS}

The differences between the results obtained using track-induced clustering and traditional methods are small (Detector 1 in Fig. 2) if the detector under test is well within its operational conditions. The differences are dominated by the choice of cluster width (TIC) and thresholds (SNR method). In the region of low SNR, the traditional methods overestimate the DUT performance.

\section{A. Cluster Width}

A small TIC cluster width tends to underestimate the collected charge. If the cluster width parameter is smaller than the true cluster width, the signal from the edges of the true cluster is not included in the reconstructed cluster. In addition, the highest signal strips of the true cluster might not be included in the reconstructed cluster if the difference between the impact points of the reconstructed and the true track is large. 


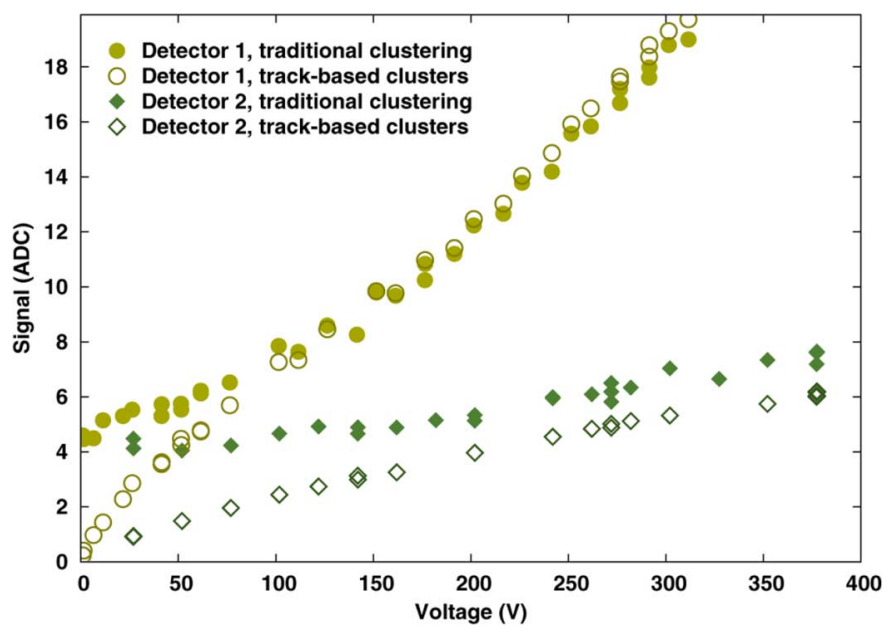

Fig. 2. Traditional and TIC method applied on the example data [1].

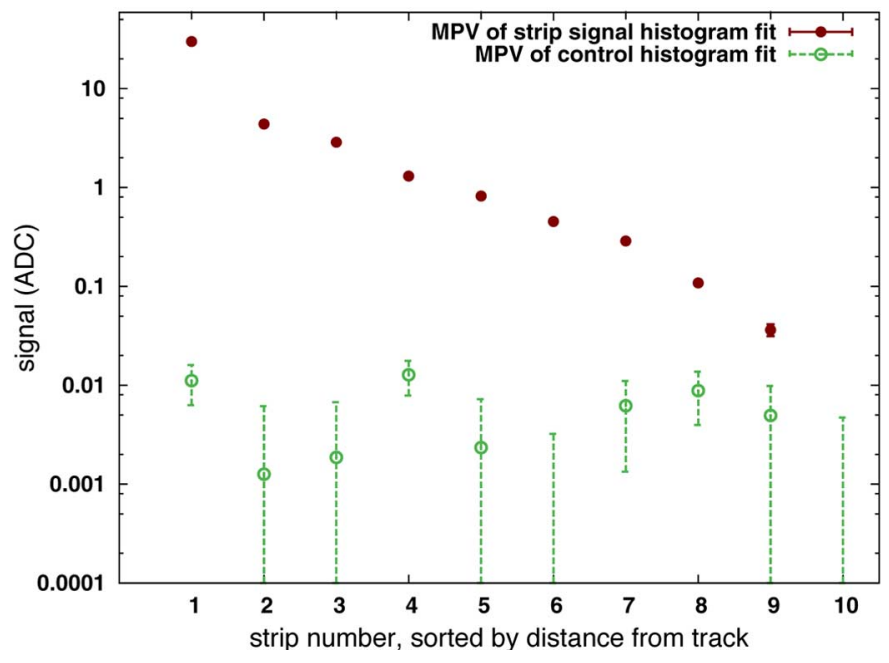

Fig. 3. Collected charge as a function of distance from track location. The data point at 1 represents the most probable value (MPV) of signals from the closest strip to the track, point at 2 shows shows the MPV of the signal from the next closest strip to the track and so on. The control plot data are generated in the manner described in Section IV-A.

Especially in case of a wide cluster, TIC can measure exhaustively the total collected charge (Fig. 3). The signal values obtained closely follow those of a non-segmented diode test setup similar to [11] and are larger than what can be achieved with the traditional methods. With a very wide cluster width the results might no longer be comparable with the traditional clustering methods, where, typically, the strips far from the peak strip are not found and are not useful for position measurement.

\section{B. Low SNR}

When the signal-to-noise level is low, there is a significant difference between the results of the clustering and TIC methods (Fig. 4 \& Detector 2 in Fig. 2). In these conditions, the distribution of the signal measured using the traditional method no longer follows the expected distribution as a result of a significant fraction of the genuine signal being left out.

A clustering method uses SNR thresholds to define clusters. Therefore the lower limit of the possible cluster signal values de-

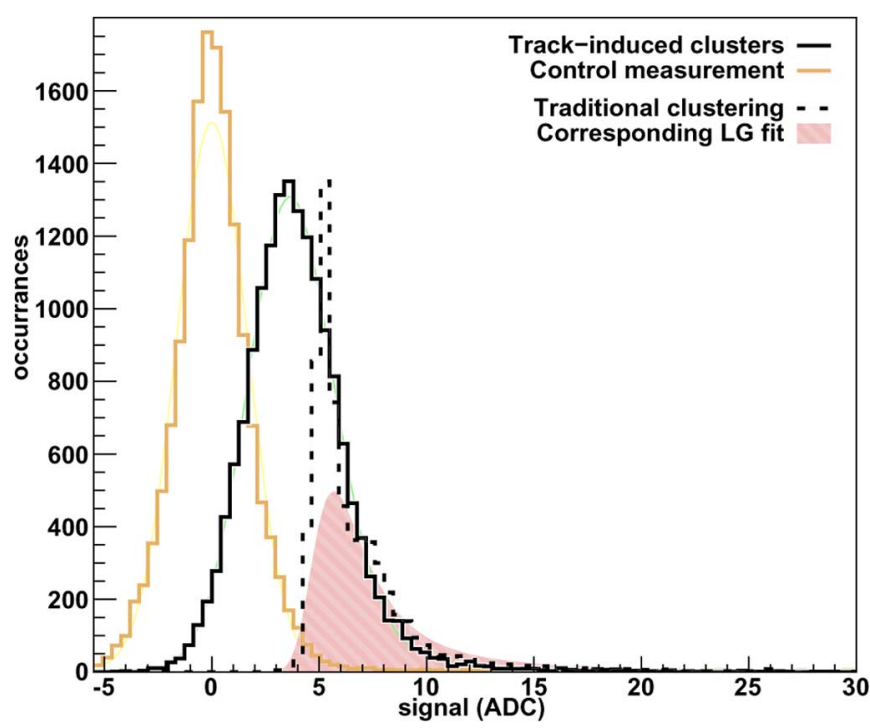

Fig. 4. The cluster charge histograms acquired using traditional and track-induced methods differ when the detector is not in its operational range. In this example the convoluted Landau and Gaussian function (LG) fits poorly to the data acquired using the traditional method. The control measurement, described in Section IV-A, is also shown.

pends on the noise levels of the individual strips but can also depend on the cluster width. The varying discrimination threshold translated into strip signal sum modifies the shape of the low-end of the signal histogram. As a result, the measured distribution might appear to follow the expected distribution even if the data are biased. Therefore it is not always possible to evaluate the success of an acquired low-SNR fit result by studying histograms acquired using traditional clustering only. Track-induced clustering analysis avoids this possibility of the systematic acquisition of overestimated signal values in detector characterization and should be used systematically to cross-check the validity of the results of the traditional method.

\section{DISCUSSION}

The use of track-induced clustering requires the track position in the local co-ordinate system of the DUT to be known. Trackbased alignment is usually required to reach this goal. The use of TIC also requires the non-zero suppressed raw analog data of the DUT to be available. If the track positional error is small compared to the DUT segment size, the TIC cluster width can be tuned to match that of a typical traditionally reconstructed cluster. If the positional error of the track is not negligible, then the TIC clusters should be at least twice as wide as the track error.

Detector 1 in Fig. 2 represents a typical case where the DUT is performing relatively well. In a barely biased case the signal acquired using traditional clustering saturates around $5 \mathrm{ADC}$ units, but there is no real risk of the results being misinterpreted. When a high enough bias voltage is applied to detector 1, the TIC method produces slightly larger signal values since a larger fraction of the total collected charge is seen. While the signal values obtained using traditional clustering for detector 2 in Fig. 2 start to increase for the highest detector bias values, they are still not correct. Drawing conclusions where the charge collection 


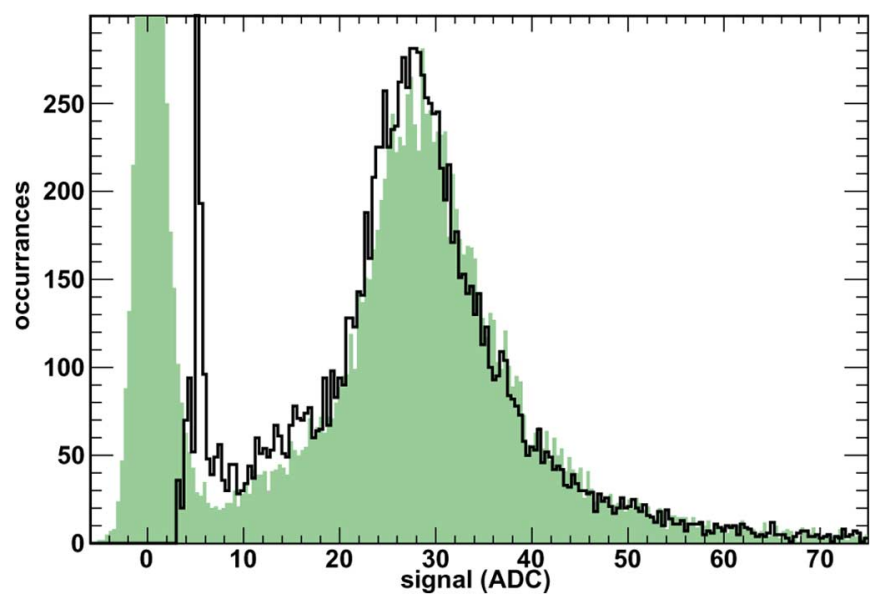

Fig. 5. Cluster charge histograms acquired using traditional (black line) and track-induced (filled graph) methods are essentially the same when the detector is in its operational range.

is claimed to be above a threshold would be dangerous in the case of detector 2 if only traditional clustering data were considered. When the signal-to-noise level of the DUT is low, there is a significant overlap between the distributions of the particle-induced charge and background noise. This is well depicted in Fig. 4, where the distribution of the TIC signals is presented, together with a control measurement. In this situation, traditional clustering methods would not be able to discriminate well between real and noise clusters (both efficiently and purely at the same time). TIC benefits from track information and is a good discriminator in these conditions.

Some practical problems are shown in Fig. 5, where a Gaussian peak lies near zero in the TIC signal histogram. This peak is caused by readout issues and the tail between the two peaks is due to charge-sharing between working and non-working segments. As the peak does not reflect the DUT response, the corresponding events should be filtered out in a careful study of the detector properties.

The plots illustrating the behavior of the TIC shown in this paper were acquired using Magnetic Czochralski silicon strip detectors in a setup [12], where the reference track uncertainty is below $10 \%$ of the DUT segment size. All the example data utilizing the TIC plotted here contain two strips wide clusters (Fig. 3 excepted). The TIC method might be suitable for the characterization of other types of position-sensitive detectors, assuming that the requirements mentioned earlier are met.
The total collected charge and the collected charge used for position measurement are distinct quantities. As traditional cluster charges are de facto standards in silicon strip beam test result reporting, one should keep this difference in mind when comparing TIC results to other beam test results.

\section{CONCLUSION}

Track-induced clustering allows the unbiased analysis of the signal and noise responses of poorly-performing position sensitive detectors. It complements the traditional analysis by avoiding several pitfalls, which play an important role especially in the region of low SNR. Track-induced analysis cannot be used to analyze properties such as efficiency and specificity, since these properties are related to the applied clustering method, and are not properties of the DUT alone.

\section{REFERENCES}

[1] P. Luukka et al., "Test beam results of heavily irradiated magnetic Czochralski silicon strip detectors," Nucl. Instrum. Methods Phys. Res. A, vol. A612, pp. 497-500, 2010.

[2] M. J. Kortelainen, T. Lampén, H. Moilanen, and T. Mäenpää, “Offline calibration and data analysis for the silicon beam telescope on the CERN H2 beam," Nucl. Instrum. Methods Phys. Res. A, vol. A602, pp. 600-606, 2009

[3] P. Fischer, R. Hammarström, S. Menke, B. Raith, O. Runolfsson, and N. Wermes, "Performance of a beam telescope using double sided silicon microstrip detectors," Nucl. Instrum. Methods Phys. Res. A, vol. A364, pp. 224-230, 1995.

[4] C. Colledani, W. Dulinski, R. Turchetta, F. Djama, A. Rudge, and P. Weilhammer, "A submicron precision silicon telescope for beam test purposes," Nucl. Instrum. Methods Phys. Res. A, vol. A372, pp. 379-384, 1996.

[5] C. Eklund et al., "Silicon beam telescope for CMS detector tests," Nucl. Instrum. Methods Phys. Res. A, vol. A430, pp. 321-332, 1999.

[6] C. Amsler et al., "A high resolution silicon beam telescope," Nucl. Instrum. Methods Phys. Res. A, vol. A480, pp. 501-507, 2002.

[7] L. A. T. Bauerdick et al., "Beam test of silicon strip sensors for the zeus micro vertex detector," Nucl. Instrum. Methods Phys. Res. A, vol. A501, pp. 340-358, 2003.

[8] D. Sacco, A. Bulgheroni, F. Risigo, and A. Baroncelli, "Using an electron beam energy scan to characterise a silicon strip telescope," Nucl. Instrum. Methods Phys. Res. A, vol. A598, pp. 675-680, 2009.

[9] M. Schieber et al., "Radiation-hard polycrystalline mercuric iodide semiconductor particle counters," Nucl. Instrum. Methods Phys. Res. A, vol. A410, pp. 107-110, 1998.

[10] A. Zoboli et al., "Functional characterization of 3D-DDTC detectors fabricated at FBK-irst," in Proc. Nuclear Science Symp. Conf. Rec., Oct. 2008, pp. 2721-2726.

[11] H. Hoedlmoser, M. Moll, M. Koehler, and K. Nordlund, "CCE measurements and annealing studies on proton-irradiated p-type $\mathrm{MCz}$ silicon diodes," Nucl. Instrum. Methods Phys. Res. A, vol. A583, pp. 64-70, 2007.

[12] T. Mäenpää et al., "Silicon beam telescope for LHC upgrade tests," Nucl. Instrum. Methods Phys. Res. A, vol. A593, pp. 523-529, 2008. 